\title{
Una propuesta para la gestión sostenible de los postgrados en Ciencias naturales para el desarrollo: flexibilidad y aplicación (Nota técnica)
}

\author{
A proposal for the sustainable management of \\ natural sciences postgraduates in development: \\ flexibility and application (Technical note)
}

Tomás de Jesús Guzmán-Hernández' 


\section{Palabras clave}

Postgrado; programas de estudios bimodales y a distancia.

\section{Resumen}

En América Latina, la realidad muestra la necesidad de mejorar la calidad de la formación universitaria de los docentes e investigadores, tanto a nivel de maestría como de doctorado. El postgrado "Doctorado en Ciencias Naturales para el Desarrollo" (DOCINADE) rompe los esquemas tradicionales de los sistemas de estudios superiores y utiliza las tecnologías de la información y la comunicación, el diálogo mediado a distancia y un sistema bimodal integrado, para ofrecer a la comunidad latinoamericana una opción de estudios flexible, pertinente, racional y vinculada con su entorno.

Varias universidades públicas de países de la región ya trabajan en este postgrado, por ejemplo, Costa Rica, México, Nicaragua y Cuba, con el apoyo de otras instituciones de educación superior de Estados Unidos, Canadá y España, entre otros. El programa ofrece cuatro énfasis: Sistemas de producción agrícola sostenible, Manejo sostenible de recursos naturales, Gestión y cultura ambiental y Tecnologías electrónicas aplicadas.

La propuesta incluye el reconocimiento de la maestría académica y la continuidad en el programa de postgrado, con temas de investigación relacionados con las prioridades locales, regionales o nacionales y dirigiendo los esfuerzos hacia la sostenibilidad, los sistemas limpios de producción y la educación ambiental.

El programa ha recibido el apoyo financiero de la Asociación Nacional de Universidades e Instituciones de Educación Superior de México y el Consejo Superior Universitario Centroamericano (CSUCA) durante el periodo 200I-2005, el Ministerio de Ciencia y Tecnología (MICIT) en 2002-20I I y el Consejo Nacional de Rectores (CONARE) en 2005-20 I 3 (estas dos últimas de Costa Rica).

El postgrado fue diseñado por doctores de universidades latinoamericanas para estudiantes provenientes de la región.

Los objetivos son compartir esta experiencia, sus resultados y encontrar nuevos aliados y socios que permitan ampliar su cobertura de acción y lograr sinergias y complementariedades.

\section{Key words}

Postgraduate studies; bimodal studies, e-learning studies.

\begin{abstract}
In Latin America, reality shows the need to improve the quality of university education of teachers and researchers, both master's and doctoral. The graduate "Doctorate in Natural Sciences for Development" (DOCINADE) shattered the traditional higher education systems and uses the information technology and communication; the distance mediated dialogue and an integrated bimodal system to provide the Latin American community with a choice of flexible curriculum, relevant, rational and linked to its environment.
\end{abstract}

Several public universities in countries in the region are already working in this graduate, for example, Costa Rica, Mexico, Nicaragua and Cuba, with the support of other institutions of higher education in the United States, Canada and Spain, among others. The program offers four emphases: sustainable crop production systems, sustainable natural resource management, environmental management and culture and applied electronic technologies.

The proposal includes recognition of academic expertise and continuity in the graduate program with research topics related to local, regional, or national priorities and directing efforts toward sustainability, cleaner production and environmental education.

The program has received financial support from the National Association of Universities and Institutions of Higher Education in Mexico and the Central American University Higher Council (CSUCA) during the 200I-2005 periods, the Ministry of Science and Technology (MICIT) in 2002-20II and the National Council of Rectors (CONARE) in 2005-20I3 (the latter two of Costa Rica).

The graduate was designed by doctors of Latin American universities for students from the region.

The objectives are to share this experience, results and find new allies and partners that will expand its coverage of action to achieve synergies and complementarities. 


\section{Introducción}

La Conferencia de las Naciones Unidas sobre Medio Ambiente y Desarrollo efectuada en Río de Janeiro, Brasil, en 1992, creó un nuevo marco de trabajo para la concertación y búsqueda de soluciones para los problemas ambientales de nuestro planeta, a escala global, regional y local. En este encuentro, gobernantes, empresarios y representantes de la sociedad civil suscribieron acuerdos básicos de trabajo que se han ido perfeccionando con el devenir de los años y en los cuales las ideas del "desarrollo sostenible" actuaron como núcleo orientador. Este Programa de Doctorado comparte la definición de desarrollo sostenible propuesto por Brooks (1990), quien plantea que "el desarrollo sostenible aspira a crear las estrategias e instrumentos que posibiliten integrar la conservación del patrimonio natural y el desarrollo, permitiendo satisfacer las necesidades humanas básicas, promoviendo la igualdad y la justicia social, la autodeterminación social, la diversidad cultural y la preservación de la integridad de los ecosistemas", citado por Guzmán (2009). Los acuerdos de la Conferencia de Río de Janeiro mostraron una comprensión cada vez más clara de que la base de recursos del planeta está sometida a una elevada tasa de erosión, que pone en peligro la supervivencia de un gran número de especies, incluyendo la especie humana, al igual que la estabilidad funcional del planeta.

En el caso de Costa Rica, país de gran tradición agrícola, la actividad agropecuaria es una fuente importante de divisas y representa uno de los principales ingresos económicos para muchas familias, cooperativas y comunidades rurales; asimismo, es objeto de estudio en varias universidades, que gradúan profesionales a nivel de grado y postgrado en las áreas de Agronomía, recursos naturales, medio ambiente y tecnologías aplicadas.

La participación relativa del sector agropecuario en el Producto Interno Bruto (PIB) permanece estable entre el $18 \%$ y el $20 \%$, y ocupa actualmente el tercer lugar en el país, después de las actividades industrial, comercial y de servicios.

El comportamiento de la actividad agropecuaria mantiene una relativa estabilidad en cuanto a la producción de cultivos tradicionales, siendo los principales rubros el cultivo de café y banano, la ganadería de carne y leche y las raíces y tubérculos, que han ganado un espacio importante como bienes de exportación.

En los últimos años el desarrollo económico del país ha sido impactado de manera muy positiva por el sector turismo, sobre todo el de tipo ecológico. Esto ha sido posible por el desarrollo de un sistema de áreas silvestres protegidas y el trabajo de investigación y docencia de las universidades estatales, que ofrecen varias carreras de grados y postgrados en estos campos.

Los recursos naturales no solo proveen bienes a la sociedad sino también servicios vitales como los ambientales, área en la cual el país deberá mantener su papel protagónico a nivel internacional. Costa Rica tiene una capacidad de uso de la tierra forestal (sin incluir los recursos de protección) de entre 23\% y $25 \%$ de su territorio.

Las diferentes actividades económicas que se realizan en el país y en la región centroamericana requieren la capacitación de profesionales con un alto grado científico. A estos se les debe preparar en el uso de las nuevas tecnologías de producción, transformación de productos, incorporación de mayor valor agregado, conservación y educación en medio ambiente, cuidado y preservación de la biodiversidad, búsqueda de soluciones a los problemas económicos con una nueva visión de mercadeo y aplicación de las nuevas tecnologías de la informática y la electrónica.

Es por todo ello que las universidades, como agentes de cambio, participan en un programa que, mediante la experiencia en la aplicación de tecnologías para la educación bimodal, utiliza el diálogo mediado a distancia, compartiendo virtualidad con presencialidad en un sistema combinado ó híbrido de enseñanza-aprendizaje.

La Universidad Estatal a Distancia de Costa Rica (UNED) cuenta con sistemas de estudio a distancia que actualmente utilizan las plataformas Moodle (software libre) y Blackboard (software con licencia), videoconferencias, audioconferencias, portales para investigación y sitios web. La Universidad Nacional de Heredia (UNA) tiene experiencia en la aplicación de la enseñanza a distancia con las maestrías en Desarrollo Rural y Tecnología Educativa, que aplican una metodología mixta utilizando medios virtuales y presenciales; además, se está consolidando el 
proyecto INNOVAR. En el Instituto Tecnológico de Costa Rica (ITCR) existe un proyecto digital llamado TEC-Virtual apoyado por Microsoft y se trabaja en otro proyecto de virtualización de actividades docentes y administrativo-académicas.

Las universidades participantes en el programa de postgrado cuentan con un desarrollo importante de infraestructura (laboratorios, bibliotecas, bases digitales de datos, fincas experimentales, publicaciones periódicas, etc.), para apoyar las investigaciones y pasantías de los aspirantes a doctorados y maestrías.

La apertura de un espacio de este tipo en los países de Mesoamérica ha generado nuevas expectativas, donde la igualdad de condiciones con otras universidades de la región podría contribuir a la creación de nuevos énfasis del programa de doctorado, identificando debilidades y fortalezas que permitan abordar nuevas áreas prioritarias, generar una masa crítica de especialistas para promover el desarrollo, a través de las actividades agroindustrial, agroempresarial, forestal, de educación y gestión ambiental, la ingeniería ambiental y la electrónica aplicada, entre otras. América Latina está en disposición real de ofrecer hoy en día programas de esta naturaleza con el apoyo de sus propios doctores a través de redes de enseñanza conjuntas e integradas.

\section{Antecedentes}

El Programa de Doctorado en Ciencias Naturales para el Desarrollo surge como una necesidad de las universidades latinoamericanas para mejorar la formación académica de docentes y profesionales de la región a nivel doctoral, utilizando las nuevas tecnologías de la información y la comunicación.

La cronología de aprobación de este programa es la siguiente: durante el periodo 200 I-2004 recibió financiamiento a través de cuatro convocatorias de la Asociación Nacional de Universidades e Instituciones de Educación Superior (ANUIES) Consejo Superior Universitario Centroamericano (CSUCA) presentado por la Universidad Autónoma Chapingo (UACh) y el ITCR, la UNA, la UNED y la Universidad Nacional Autónoma de Nicaragua en León (UNAN-León-. En 2003 se integra la Universidad Nacional Autónoma de México (UNAM) a través de la Facultad de Estudios
Superiores Cuautitlán (FES, Cuatitlán). Ese mismo año, se integra el Consejo Nacional de Rectores (CONARE) de Costa Rica, como el primer programa de Doctorado interuniversitario latinoamericano, inter, trans y multidisciplinario.

En todos los años de trabajo entre 2002 y 2009, El Ministerio de Ciencia y Tecnología de Costa Rica (MICIT) ha apoyado con financiamiento para el transporte y manutención de académicos de México, Nicaragua, Cuba y España. Este apoyo económico se ha utilizado para organizar seminarios y talleres internacionales en Costa Rica y Nicaragua desde 2005 hasta 2009.

En 2005 se inició de manera oficial el programa de postgrado interuniversitario Doctorado en Ciencias Naturales para el Desarrollo (DOCINADE), en la sede del CONARE, en el edificio Franklin Chang en San José.

Una vez aprobado el doctorado, el equipo de académicos se dió a la tarea de elaborar y diseñar un Programa vinculado al proyecto Alfa II, de la Unión Europea, que constituyó una nueva propuesta de postgrado de la Maestría Internacional en Gestión de los Recursos Naturales para el Desarrollo Sostenible.

Esta propuesta se aprobó como Proyecto Alfa II 0396-A, denominado "Programa Interuniversitario de postgrado On -line euro- latinoamericano en manejo y conservación de recursos naturales, sistemas agropecuarios sostenibles y medio ambiente para el desarrollo" y se desarrolló entre los años 2005-2007.

\section{El sistema interuniversitario de Estudios de Postgrados "Doctorado en Ciencias} Naturales para el Desarrollo"

En la figura I se puede observar este sistema interuniversitario en Costa Rica, con sus correspondientes características académicas:

Excepcionalmente, se podría lograr la incorporación de profesionales con grado de licenciatura al doctorado, en aquellos casos que demuestren un gran bagaje en investigaciones y en publicaciones en su trayectoria laboral. 
Figura I. En los postgrados a nivel de maestrías se puede matricular a partir de los grados de bachillerato y licenciatura, y con grado de maestrías el de doctorado.

Sistema Universitario de Estudios de Postgrados en el DOCINADE

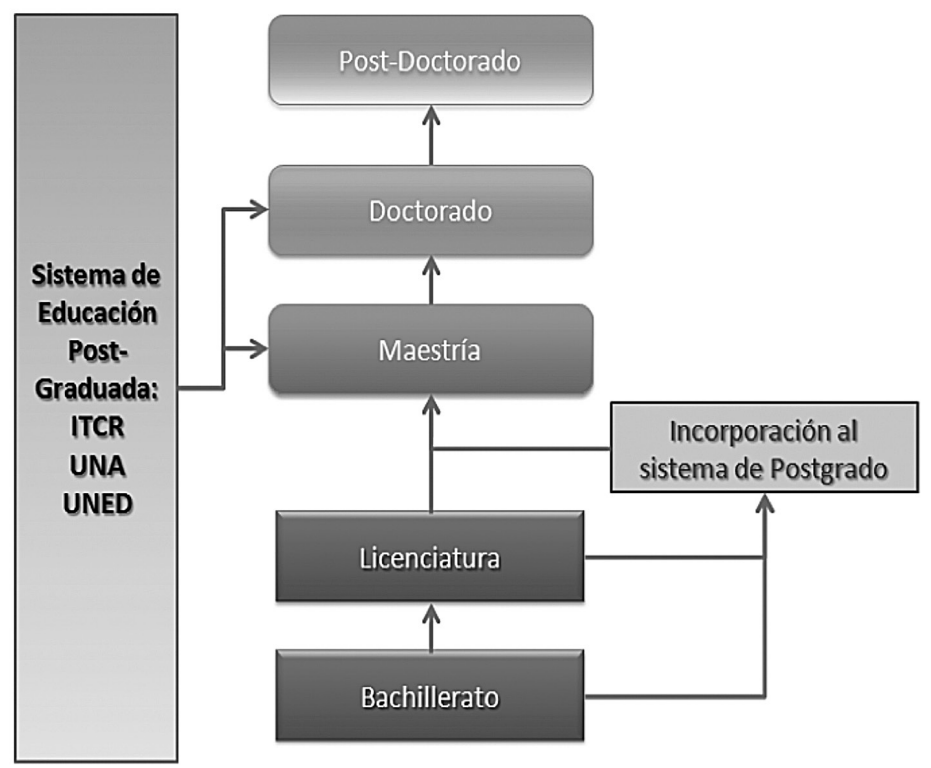

\section{Las actividades académicas}

En el DOCINADE, las actividades académicas son: el eje central, las investigaciones (básicas o aplicadas), la actividad curricular de formación del investigador definido por cursos obligatorios y optativos, una pasantía en una institución externa al aspirante, el examen de candidatura, la presentación del documento de tesis y su defensa.

Esta estructura se puede observar en la figura 2.

\section{Características del programa}

Las características que acompañan a este plan de estudios y que lo convierten en un programa educativo único en la región latinoamericana son las siguientes: cuatro años de duración ( 100 créditos), integración de universidades, facultades, escuelas y centros de investigaciones vinculadas al campo del doctorado y la maestría que se está gestando, como un programa directo y de investigaciones académicas; flexibilidad curricular y metodológica (programa bimodal: presencial, semipresencial, con fuertes componentes virtuales y a distancia); coordinación colegiada, desarrollo de cuatro énfasis vinculados con la actividad agrobiológica, ambiental y tecnológica, congruente con la misión de cada universidad participante; diseño del plan de estudios según intereses particulares de las necesidades laborales de los estudiantes, pertinencia y calidad, vinculación directa con proyectos internacionales tales como:

Figura 2. Estructura básica de las actividades académicas del DOCINADE

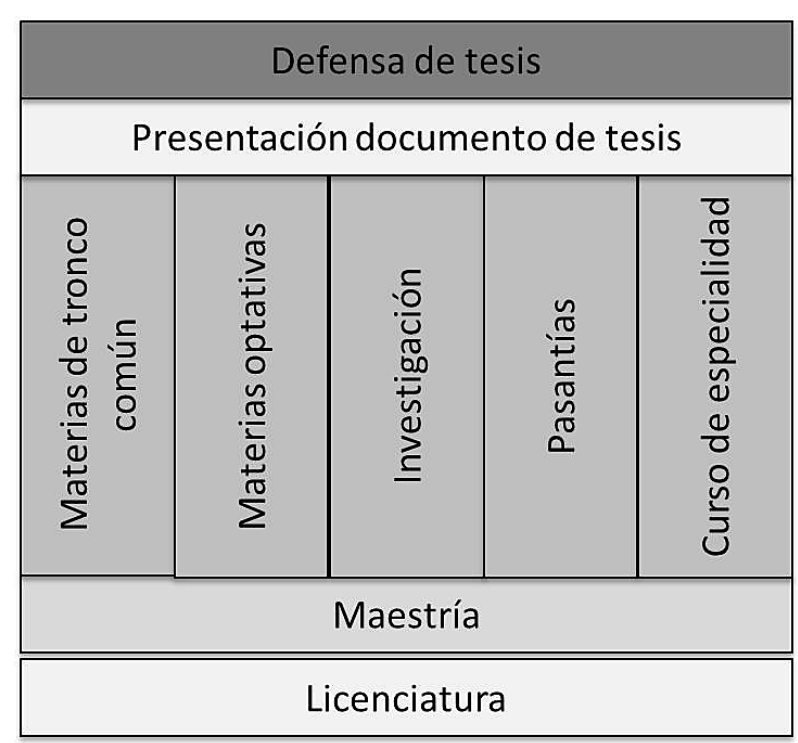


"Programa Interuniversitario de postgrado On -line euro- latinoamericano en manejo y conservación de recursos naturales, sistemas agropecuarios sostenibles y medio ambiente para el desarrollo": Proyecto Alfa II 0396-A, UE-AL y con la propuesta recientemente aprobada por los gobiernos de Costa Rica y México cuyo título es: "Postgrado mesoamericano interuniversitario conjunto Costa Rica-México:capacitación, preparación metodológica, didáctica y pedagógica de las asignaturas en el sistema bimodal: maestría y doctorado en ciencias naturales para el desarrollo sostenible".

La idea básica del diseño de este programa ha sido la integración de América Latina y especialmente de Centroamérica, con el apoyo de instituciones de la región y con la participación internacional abierta.

\section{Población meta del programa}

Este postgrado está específicamente dirigido a profesionales dispuestos a estudiar utilizando las novedades de las tecnologías de la información y la educación a distancia. Los profesionales que se matriculen deben tener interés en retransmitir a otros sectores de la población la necesidad de producir y capacitarse utilizando técnicas que permitan el desarrollo sostenible, la aplicación de tecnologías limpias y la equidad de género.

El postgrado bimodal o híbrido está orientado en primer término a profesionales de universidades, empresas y de otras instituciones de América Latina, el Caribe y Europa, así como a cualquier otra persona interesada en las áreas de acción definidas anteriormente.

El grado que deberán tener las personas que deseen cursar este postgrado es el de licenciatura de manera excepcional o el título universitario de Maestría profesional o académica.

Por otra parte, se dirige a una población meta que por razones laborales, de tiempo, económicas o de ubicación de los actuales postgrados de maestría y de doctorado, nacionales o en el extranjero, no puede matricularse en dichos postgrados.

Los estudiantes que se matriculen deben contar como mínimo con acceso a computadoras, internet, salas para videoconferencias y otras facilidades de conexión y conectividad, en sus respectivas localidades.

\section{Ejes curriculares del programa}

Los cursos optativos y obligatorios, la preparación y evaluación del curso de especialidad de cada énfasis, así como la investigación tanto del proyecto de tesis como en cada curso, se basarán en los siguientes ejes curriculares, entendidos como actividades formativas y metodológicas, que dan soporte como elementos integradores y dinamizadores a través de todo el plan de estudios:

- Investigación. Desde que se matricula en el programa, el aspirante enfoca todo su quehacer dentro del plan de estudios a la investigación, caracterizada por el rigor y excelencia, que conduzca a la generación de nuevos aportes al conocimiento cientííco.

- Sostenibilidad y desarrollo. Este eje curricular pretende abordar lo concerniente al desarrollo económico y social del ser humano, la conservación del medio ambiente, el uso racional de los recursos naturales, la mejora de la competitividad en la economía local y global, el respeto a los derechos humanos, a la diversidad étnica, cultural y de género, así como el mejoramiento de las relaciones sociales y de la calidad de vida.

- Gestión estratégica de la información y la tecnología. Este eje comprende el desarrollo de la capacidad para lograr el acceso y manejo de la información.

\section{Los énfasis del programa}

Dada la experiencia acumulada y la formación profesional de los académicos de las instituciones participantes, se ofrecen los siguientes énfasis, a saber:

- Sistemas de Producción Agrícola

- Gestión de Recursos Naturales

- Gestión y Cultura Ambiental

- Tecnología de electrónicas aplicadas

La Figura 3 muestra las características generales del programa, incluyendo los ejes curriculares, los énfasis y las áreas temáticas o disciplinarias, donde se insertan las tesis de nuestros estudiantes.

\section{Opción metodológica}

La modalidad pedagógica de este programa es el diálogo mediado a distancia, utilizando las nuevas 
Figura 3. Características generales del programa.

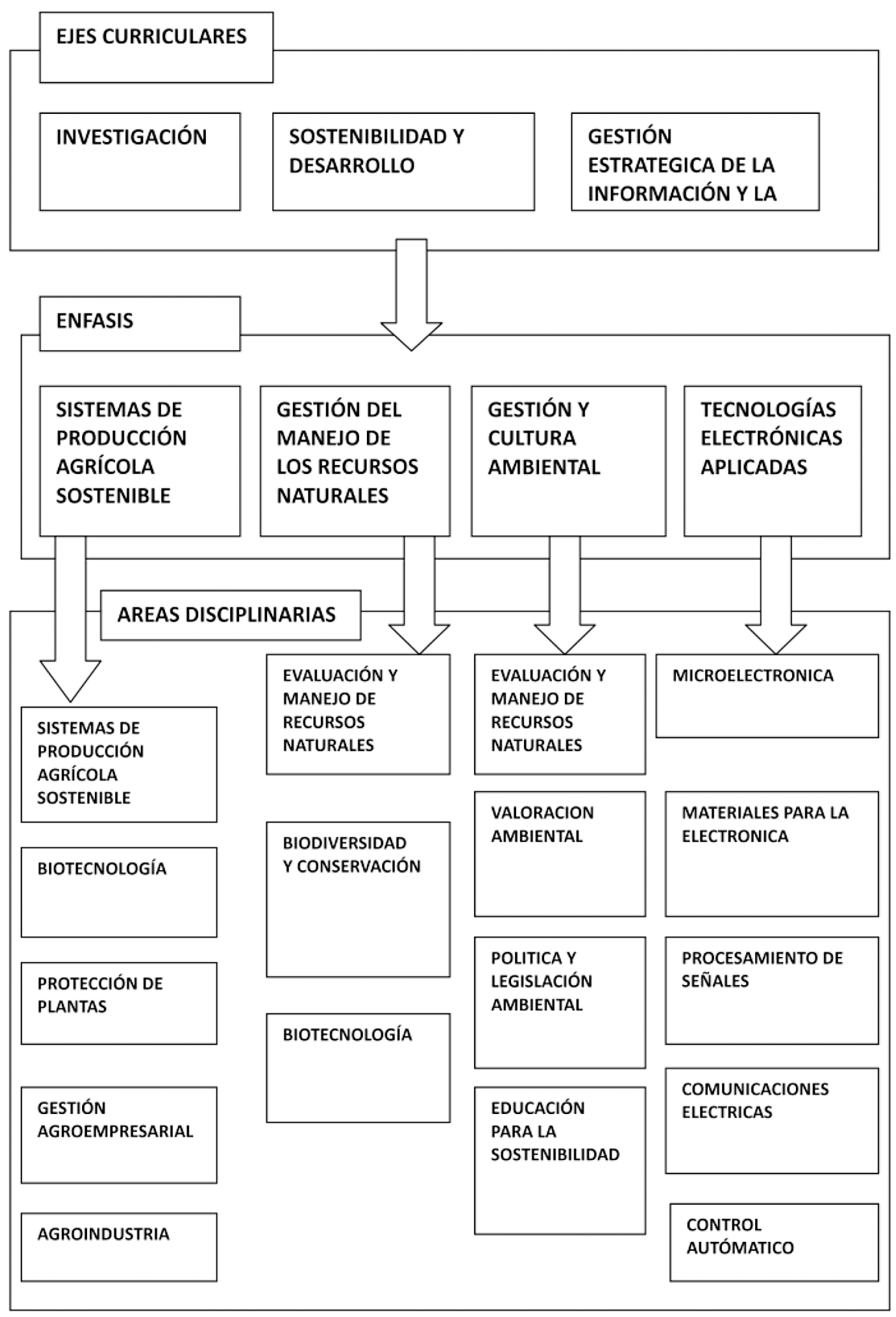

tecnologías de la comunicación y la información, y actividades presenciales cuando las condiciones del postgrado así lo requieran. Para ello, las universidades participantes disponen de la infraestructura necesaria para implementar esta modalidad. El programa está orientado a formar profesionales capaces de apropiarse de las estrategias cognitivas y destrezas en el área de los recursos naturales, desarrollando comprensión y actitud de tolerancia ante la diversidad cultural y regional, utilizando la tecnología como un medio para obtener, confrontar y producir conocimientos. 
El modelo educativo es bimodal y esto incluye presencialidad, estudios a distancia, virtualidad y trabajo on-line, así como la comunicación virtual a través de plataformas e-learning, entre otras formas de comunicación y conexión.

Las instituciones universitarias participantes forman un sistema social abierto, en el cual se pasa de una formación tradicional a otra que está focalizada en el contexto y en el propio aprendizaje del alumno. Este tipo de organización se caracteriza por su asimetría, regionalización, diversificación y capacidad para generar conocimiento, codificarlo, conservarlo y ponerlo a disposición de sus miembros y otras comunidades externas, para su respectiva transferencia a otros usuarios.

El modelo educativo que sustenta los procesos virtuales exige principalmente dos herramientas: los sistemas hipermedia, como forma de estructurar la información; y las redes de comunicación de área extendida, como soporte de la información. Es decir, la red Internet y la red de Internet avanzado o Internet dos. Es importante resaltar que una institución universitaria que pretenda desarrollar un aprendizaje utilizando este modelo necesita una estructura particular, o una "cibercultura" de todos sus miembros, ya que este implica primero tener conciencia y disposición al cambio de sistemas, ya que la construcción de este nuevo paradigma requiere de una interacción permanente: HombreSociedad-Ambiente y la apropiación de los fundamentos teórico-metodológicos que sustentan cada universidad e institución participante de la Red, con la sociedad, el entorno y la investigación cientííca.

\section{Plan de estudios del doctorado}

El Plan de Estudios Doctoral comprende un total de 100 créditos, distribuidos en una fase teórica y una fase de investigación. La fase teórica tiene un total de 33 créditos y la fase de investigación de 67 créditos.

\section{Tronco común (asignaturas obligatorias)}

Comprende:

- Cursos del tronco común (I 8 créditos).

- Seminario - Taller de investigación I, II, y III (I7 créditos).

\section{Cursos optativos según énfasis}

Estos son cursos propios del énfasis correspondiente, para garantizar la profundidad de los conocimientos en cada uno. El estudiante elegirá 3-4 cursos en correspondencia con el énfasis seleccionado, para un total de 10 créditos.

\section{Curso de especialidad}

El curso de especialidad (5 créditos), que concluye con la presentación del examen de candidatura, tiene el objetivo de que el aspirante pueda demostrar el dominio sobre el tema relacionado con el énfasis seleccionado y con sus estudios de tesis.

\section{Trabajo de tesis}

El trabajo de investigación incluye los cursos de Investigación de tesis I al $\mathrm{V}$, la defensa de la tesis y su presentación ante un tribunal. El total de créditos en investigación directa es de 40, más los 17 créditos de los seminarios talleres obligatorios I, II y III, lo cual da un total de 57 créditos en investigación.

\section{Pasantía}

Cada aspirante debe cursar una pasantía corta (entre 3 y 6 meses) en algún centro de investigación de su país o del extranjero (que no sea su centro de trabajo) con un total de 10 créditos.

Para la ejecución del Programa de Doctorado se contempla una fase previa de preparación y capacitación en el uso de las tecnologías virtuales que se aplicarán. En esta actividad participan tanto docentes como estudiantes.

Este programa se propone ofrecer además una Maestría académica, que surge del proyecto Alfa II 0396-A, directamente relacionada con el Doctorado, y cuya estructura es la siguiente:

\section{Plan de estudios de la maestría internacional actualmente en trámite de aprobación}

La estructura y mapa curricular se puede observar en la figura 4.

Como indica el diagrama anterior, los cursos de la maestría se ubican en tres niveles: Tronco común: 
Figura 4. Plan de estudios del DOCINADE. Fuente: Elaboración del equipo de trabajo.

Maestría Internacional en Gestión de los Recursos Naturales para el Desarrollo Sostenible

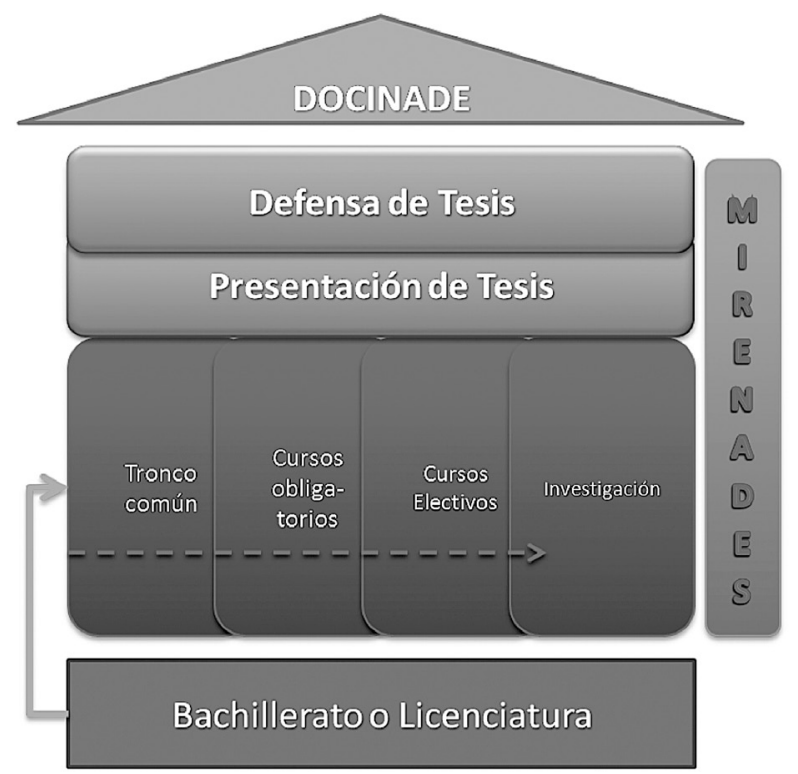

comunes y obligados para todos los énfasis; énfasis: obligatorios y específicos para cada énfasis; electivos: el estudiante los selecciona de acuerdo con sus intereses; investigación de tesis, trabajo de tesis.

Los participantes en la maestría podrán elegir el énfasis en función de sus necesidades y del mercado laboral y a su vez seleccionar los cursos electivos de acuerdo con los requerimientos de su formación.

\section{Cursos del tronco común}

Estadística aplicada, Desarrollo sostenible:Fenómenos globales y efectos locales, Problemática ambiental y Gestión de los recursos naturales y Metodología de la investigación, entre otras materias.

\section{Aspectos generales del programa}

Dosaños de duración ( 120 créditosECTS para Europa y 67 créditos para América Latina); la integración de las fortalezas académicas de universidades, facultades, escuelas y centros de investigaciones vinculadas al objeto de estudio de esta maestría; flexibilidad curricular y metodológica (Programa bimodal: presencial con fuertes componentes virtuales); coordinación colegiada, mediante un consejo de gestión académica interuniversitario e internacional; vinculación con el DOCINADE, Convenio ITCR-
UNA-UNED en Costa Rica; desarrollo de cinco áreas vinculadas con la gestión sostenible de los recursos naturales; congruencia con la misión y visión de cada universidad participante; pertinencia y calidad.

\section{Población meta}

El postgrado "Maestría Internacional en Gestión de los Recursos Naturales para el Desarrollo Sostenible" a distancia está orientado a profesionales de universidades, empresas y otras instituciones de América Latina y el Caribe, así como para cualquier otro interesado en las áreas de acción definidas en el postgrado.

El grado que deberán tener las personas que deseen cursar esta maestría es el de bachillerato universitario o licenciatura.

\section{Resultados del programa de Doctorado 2005-20II}

Las convocatorias del programa se realizan cada dos años, con un año de anterioridad y de manera rotativa entre las universidades costarricenses y con la participación activa de universidades de México, Nicaragua y Cuba. 
De esta manera, hasta la fecha se han realizado seis convocatorias ordinarias: en los años 2005, 2010 y 201 I en el ITCR; en 2007 y 2013 en la UNA, en 2009 en la UNED. La séptima convocatoria del programa será en el año 2015 en la UNED.

\section{Estado actual del programa}

De acuerdo con lo anterior, la situación actualizada del programa se presenta en los cuadros que se muestran a continuación.
Los graduados en general están distribuidos de la siguiente manera: en el ITCR I4, en la UNA 6, para un total de 20 graduados; y de acuerdo con los énfasis son 10 en Gestión y Cultura Ambiental, 6 en Manejo de Recursos Naturales y 4 Sistemas de Producción Agrícola.

Los estudiantes participantes en el programa pertenecen a los siguientes países: Costa Rica, Nicaragua, Honduras, Panamá, Guatemala, México, República Dominicana, Venezuela, Perú, Colombia, Bolivia, España, Holanda y Suiza.

Cuadro I. Estado actual del programa

\begin{tabular}{|c|c|c|c|c|c|c|}
\hline Institución/año & 2005 & 2007 & 2009 & 2010 & 2011 & 2013 \\
\hline ITCR & 4 & & & 12 & 26 & \\
\hline UNA & & 19 & & & & 20 \\
\hline UNED & & & 27 & & & \\
\hline $\begin{array}{l}\text { Total real } \\
\text { ITCR-UNA-UNED }\end{array}$ & \multicolumn{6}{|c|}{108} \\
\hline Graduados hasta 2013 & \multicolumn{6}{|c|}{20} \\
\hline
\end{tabular}

Cuadro 2. Participación de hombres y mujeres en los énfasis, así como de los países.

\begin{tabular}{|c|c|c|c|c|c|c|c|c|}
\hline \multicolumn{9}{|c|}{ TOTAL GENERAL DOCINADE } \\
\hline Género & $N^{\circ}$ & Países & $N^{\circ}$ & Énfasis & $N^{\circ}$ & $\begin{array}{l}\text { Énfasis por } \\
\text { género }\end{array}$ & Mujeres & Hombres \\
\hline \multirow[t]{2}{*}{ Mujeres } & 35 & Costa Rica & 52 & $\begin{array}{l}\text { Gestión y Cultura } \\
\text { Ambiental }\end{array}$ & 34 & GCA & 11 & 23 \\
\hline & & Colombia & 12 & $\begin{array}{l}\text { Gestión de Recursos } \\
\text { Naturales }\end{array}$ & 36 & GRN & 14 & 22 \\
\hline \multirow[t]{10}{*}{ Hombres } & 73 & Guatemala & 23 & $\begin{array}{l}\text { Sistemas de Producción } \\
\text { Agrícola }\end{array}$ & 23 & SPA & 7 & 16 \\
\hline & & Nicaragua & 5 & $\begin{array}{l}\text { Tecnologías Electrónicas } \\
\text { Aplicadas }\end{array}$ & 16 & TEA & 4 & 12 \\
\hline & & México & 8 & & & & & \\
\hline & & Panamá & 2 & $\begin{array}{l}\text { Graduados } 20 \text { / siendo } \\
\text { una población de } 108 \\
+20 \text { graduados de } 128 \\
\text { estudiantes }\end{array}$ & & & & \\
\hline & & Honduras & I & & & & & \\
\hline & & Puerto Rico & । & & & & & \\
\hline & & Rep.Dominicana & । & & & & & \\
\hline & & Perú & I & & & & & \\
\hline & & Venezuela & । & & & & & \\
\hline & & Bolivia & I & & & & & \\
\hline Total & 108 & 12 países & & & & & & \\
\hline
\end{tabular}


A su vez, en el cuadro 2 se observa la participación en los diferentes énfasis del programa, así como el número de hombres, mujeres y de los diferentes países de América Latina.

La retención obtenida en el programa ha sido en general muy alta, así como el crecimiento en cada generación. Los porcentajes de retención han sido de $63,3 \%, 83,3 \%, 78,57 \%$ y $81,25 \%$ respectivamente en cada generación.

En la nueva generación, que se matriculó en la UNED de Costa Rica en 2009, por primera vez se incorporaron estudiantes del área de electrónica aplicada, ya que este énfasis fue aprobado por el CONARE en 2008.

En el cuadro 3 se pueden observar los aspirantes en el programa por institución.

La participación de socios latinoamericanos e incluso nacionales es imperativa, ya que, según el reglamento, cada estudiante del programa necesita tres doctores en el comité de tesis, de los cuales uno es el director de tesis o tutor.
Las universidades nacionales miembros del programa no podrían proporcionar esta cantidad de estudiantes si no fuera por la participación de otras universidades socias $u$ otras instituciones tanto nacionales como extranjeras.

En la actualidad hay directores de tesis de Costa Rica, México, Nicaragua, Colombia, Bolivia,Venezuela, Canadá, Estados Unidos, Holanda, Inglaterra, Francia y España, un total de 12 países, lo cual implica un gran nivel de coordinación con ellos de manera sistemática.

\section{Estudiantes becados a nivel nacional e internacional}

En cada generación, el Comité de gestión académica del programa ofrece a la comunidad nacional e internacional al menos seis becas, para que funcionarios de las universidades públicas de Costa Rica y las universidades socias matriculen a profesores para obtener el título de doctor, como se muestra en el cuadro 4.

Cuadro 3. Aspirantes vigentes en el ITCR, la UNA y la UNED hasta el año 2013.

\begin{tabular}{|c|c|}
\hline Institución & $\begin{array}{c}\text { Número total actual de estudiantes en cada } \\
\text { Institución }\end{array}$ \\
\hline ITCR & 42 \\
\hline UNA & 39 \\
\hline UNED & 27 \\
\hline Total & 108 \\
\hline
\end{tabular}

Cuadro 4 Número de becados general del programa.

\begin{tabular}{|c|c|c|c|c|c|}
\hline Institución & 2005 & 2007 & 2009 & 2011 & 2013 \\
\hline ITCR & 2 & 2 & 6 & 7 & 3 \\
\hline UNA & 2 & 2 & 1 & 4 & 3 \\
\hline UNED & 2 & 1 & 1 & 1 & 1 \\
\hline $\begin{array}{c}\text { UNAN-León } \\
\text { Nicaragua }\end{array}$ & & 2 & 1 & & \\
\hline $\begin{array}{c}\text { Total de becados } \\
\text { Por año }\end{array}$ & 6 & 7 & 9 & 12 & 7 \\
\hline Becados totales & 41 & & & & \\
\hline
\end{tabular}


El número total de becados, que no tienen que financiar sus estudios, se ha incrementado con el tiempo y constituyen un total de 4 I estudiantes, de ellos 21 son costarricenses. Esto significa un aporte importante del programa al país y a América Latina. La beca consiste en la exoneración del pago de todos los créditos por un valor de US \$10000.

\section{Capacitaciones realizadas en sistemas de estudios a distancia, en entornos virtuales y sistemas bimodales}

Durante esta etapa de trabajo se han realizado las siguientes capacitaciones de estudiantes y docentes, tanto nacionales como extranjeros:

Herramienta Microcampus (plataforma e-learning de software libre, donado a la UNED por la Universidad de Alicante, España, utilizado en tres ocasiones en el programa); Herramienta Moodle (plataforma e-learning de software libre, que utiliza la UNED); Alf. (plataforma e-learning de software libre, aportado por la UNED de Madrid de manera gratuita); WebCT (plataforma e-learning licenciada aportada por la UNED al programa, pero esta universidad paga la licencia de uso para todas las universidades participantes nacionales y extranjeras); LMRN (plataforma e-learning aportada por el ITCR de manera gratuita); Blackboard de la UNED de Costa Rica y que constituye un plataforma licenciada.

\section{Vías utilizadas en el proceso de enseñanza-aprendizaje en el programa}

En el proceso de enseñanza-aprendizaje bimodal se utilizan de manera sistemática las siguientes vías de contacto, tanto con estudiantes como con docentes: encuentros presenciales obligatorios (dos veces al año), cursos en línea, plataformas e-learning, Internet universitaria, encuentros presenciales no obligatorios de los cursos en Costa Rica y otros países, videoconferencias en tiempo real, teléfono, Skype, chat por internet en sus diferentes modalidades, reuniones de estudiantes y sesiones periódicas del Comité de Gestión Académica del programa para analizar la marcha de toda la actividad docenteinvestigativa.

\section{Principales logros}

Hoy podemos mostrar los resultados obtenidos, los cuales se resumen a continuación:

- Integración de las fortalezas científicas, de recursos humanos, de infraestructura y materiales presentes en las universidades estatales para desarrollar estrategias conjuntas de formación en postgrado con alta calidad.

- Integración en este proyecto educativo de distinguidas universidades e investigadores de México, Cuba y Nicaragua, en América Latina, y de España, en Europa.

- Aprovechamiento de las nuevas tecnologías de la información y la comunicación (TIC) para facilitar el acceso a postgrados de profesionales que tienen limitaciones de carácter geográfico, laboral o familiar.

- Contar con 108 estudiantes de toda América Latina, pertenecientes a seis generaciones de aspirantes y la apertura programada de la sexta promoción para 20I3, en la UNA.

- Opiniones satisfactorias de los estudiantes por la calidad del programa recibido.

- Consolidación de un grupo académico interuniversitario cohesionado y comprometido con el desarrollo sostenible de la región, al cual se han ido incorporando nuevos académicos de prestigio a nivel nacional e internacional.

- Trabajo interdisciplinario mediante una red de cooperación nacional e internacional que trabaja con objetivos comunes.

- Es un proyecto innovador, pertinente y con calidad.

- Incorporación de nuevos doctores al programa, convencidos del trabajo hasta ahora realizado.

- Otro logro significativo en el trabajo interuniversitario del programa es la creación de un nuevo postgrado: "Maestría internacional en gestión de los recursos naturales para el desarrollo sostenible", Proyecto Alfa II 0396-A y la presentación de una nueva propuesta a la Unión Europea.

- Incorporación de docentes de universidades centroamericanas, como la Universidad de San Carlos de Guatemala. 


\section{Limitaciones}

También podemos mostrar los aspectos negativos, con los que tiene que lidiar un programa de esta naturaleza:

- Falta de comprensión y apoyo de parte de algunas autoridades institucionales dentro del sistema universitario.

- Críticas infundadas por falta de conocimiento de los sistemas aplicados.

- Poca participación de docentes-doctores nacionales e internacionales que potencialmente podrían incorporarse al grupo de trabajo del programa, que por cuestiones relacionadas con las contradicciones lógicas entre el trabajo virtual, a distancia y presencial, relacionados con la investigación científica, no lo hacen.

- Problemas relacionados con la asignación de jornadas laborales adecuadas para que los docentes puedan atender sus responsabilidades en el programa, en cada institución participante.

- Se recibe salario desigual por igual trabajo, de acuerdo con las normas internas de cada universidad.

- Muchos procedimientos administrativos que limitan el manejo ágil y flexible del programa para un mejor aprovechamiento de los recursos disponibles, debido a que las estructuras administrativas obligan a excesivos y complicados trámites que generan desgaste, frustración y cansancio innecesario.

- Éxodo de valiosos doctores que se han acogido a la jubilación y las limitaciones para su contratación, debido a mecanismos obsoletos y discriminatorios.

- Desconocimiento por parte de los doctores y el personal administrativo de los nuevos sistemas de enseñanza-aprendizaje en entornos bimodales, virtuales y en procesos a distancia.

- Resistencia al cambio de paradigma en los procesos de enseñanza a nivel de postgrado.

- En algunas unidades académicas no reconocen la legitimidad del DOCINADE, como un programa integral, básicamente por desconocimiento.

\section{Lecciones aprendidas}

Las "diferencias interuniversitarias" en reglamentos, estatutos, etc. se pueden obviar cuando se comparten realmente objetivos comunes y existe la voluntad de trabajar de manera colectiva, con respeto mutuo y perseverancia. Se debe trabajar para vincular a los jóvenes doctores a este tipo de sistema, ya que los más viejos no aceptan este cambio de paradigma por desconocimiento y temor. Se requiere la incorporación de todos aquellos doctores convencidos de hacer investigación científica y docencia por medios virtuales y a distancia. Se requiere paciencia ante la cantidad y diversidad de reglamentaciones internas universitarias nacionales e internacionales. El Coordinador general necesita una jornada de tiempo completo para atender las necesidades del Doctorado.

Este tipo de postgrado interuniversitario requiere una unidad administrativo-financiera diferente, para poder desarrollar métodos de trabajo integrales más ágiles y flexibles relacionados con la academia, es decir, la docencia de alto nivel, la investigación y la extensión universitaria. Aunque cuenta con la colaboración de universidades extranjeras, la responsabilidad de las actividades académicoadministrativas debe mantenerse en manos de las universidades nacionales a las cuales les fue aprobado el programa de postgrado.

\section{Perspectivas de futuro}

Las perspectivas de futuro del programa son las siguientes:

- Apertura de nuevas plazas a lo interno del programa en cada institución participante, en la UNA, el ITCR y la UNED, debido a que por acuerdo del CONARE, el financiamiento del FEES culminará en 2014 y a partir de este año el Programa deberá ser considerado un programa universitario permanente a nivel nacional, como parte de la oferta educativa de cada institución.

- Consolidación de la Unidad académica-administrativa del programa con sus reglamentaciones respectivas, relacionadas con la docencia, la investigación que logre la formación de doctores y la extensión.

- La firma de convenios nacionales e internacionales para darle sustento al programa, en los próximos años, con América Latina y Europa. 
- Presentación de la propuesta completa de Doctorado y Maestría a un programa Europeo de financiamiento, buscando la movilidad académica de estudiantes y profesores.

- Exposición de la nueva propuesta de la Maestría adjunta al programa, como un postgrado compartido con países de Europa.

- Presentación de la propuesta al padrón de excelencia de CONACYT de México, a través de la Universidad Autónoma Chapingo y la UNAM; así como la propuesta de título compartido con estas universidades y la iniciación del proceso de autoevaluación con vistas a la acreditación nacional y regional en Centroamérica.

La calidad del programa ha sido evaluada de positiva por los propios estudiantes en encuestas realizadas cada año (2005-20l I), durante el proceso de análisis de sus anteproyectos de tesis y de avances de investigaciones, como puede mostrarse en los siguientes figuras:

En la figura 5 se muestra la opinión de los estudiantes sobre los seminarios realizados hasta el año 201 l.

En la figura 5 se presenta la opinión de los participantes en los diferentes seminarios organizados por el DOCINADE desde sus inicios. Se observa que, en términos generales, la opinión ha sido en su mayoría excelente o muy buena, destacándose los dos seminarios realizados durante 20 I I en los cuales no hubo ninguna opinión desfavorable.

Tal como muestra la figura 6, la mayoría de los participantes en los diferentes seminarios realizados manifiesta haber recibido información oportuna acerca del seminario taller y sus contenidos, destacándose el primer seminario de 20 I l, al obtener una calificación favorable del 100\%.

En la figura 7 se presenta la evaluación de los participantes acerca del proceso de discusión en las presentaciones de avance de tesis de los años 2009, 2010 y 201 I. Esta refleja una evolución en las opiniones favorables, ya que en 2009 las calificaciones de bueno a excelente estaban por debajo de $80 \%$, en 2010 eran superiores al $80 \%$ y para 201 I las opiniones favorables fueron del 100\%.

Estos resultados están condicionados por la experiencia que han ido acumulando durante los últimos siete años los docentes participantes en el DOCINADE, de las instituciones y los países miembros del programa.

\section{Algunas áreas de trabajo de las actuales tesis del programa según énfasis}

Finalmente, a continuación se presentan los títulos de algunos de los trabajos que se desarrollan en el

Figura 5. Opinión de los participantes sobre la organización de los seminarios, el taller de inducción y los seminarios talleres presenciales obligatorios.

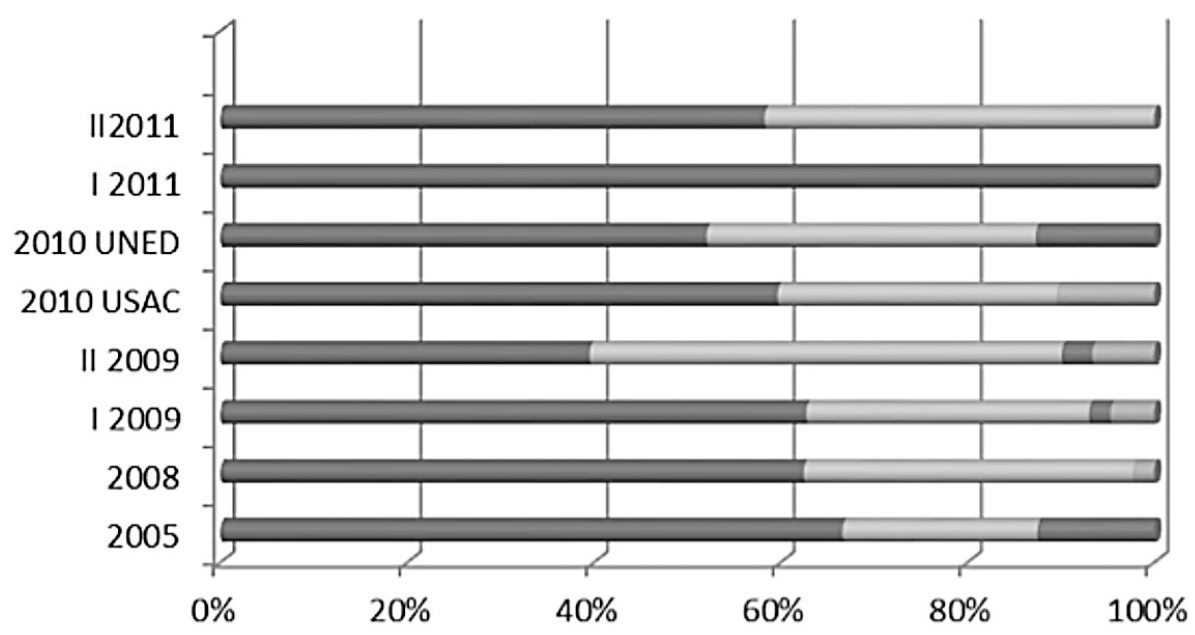

$\square$ MuyBueno $\square$ Bueno $\quad$ Regular $\square$ Deficiente 
Figura 6. Opinión de los participantes sobre la disponibilidad de información oportuna sobre el seminario taller y sus contenidos.

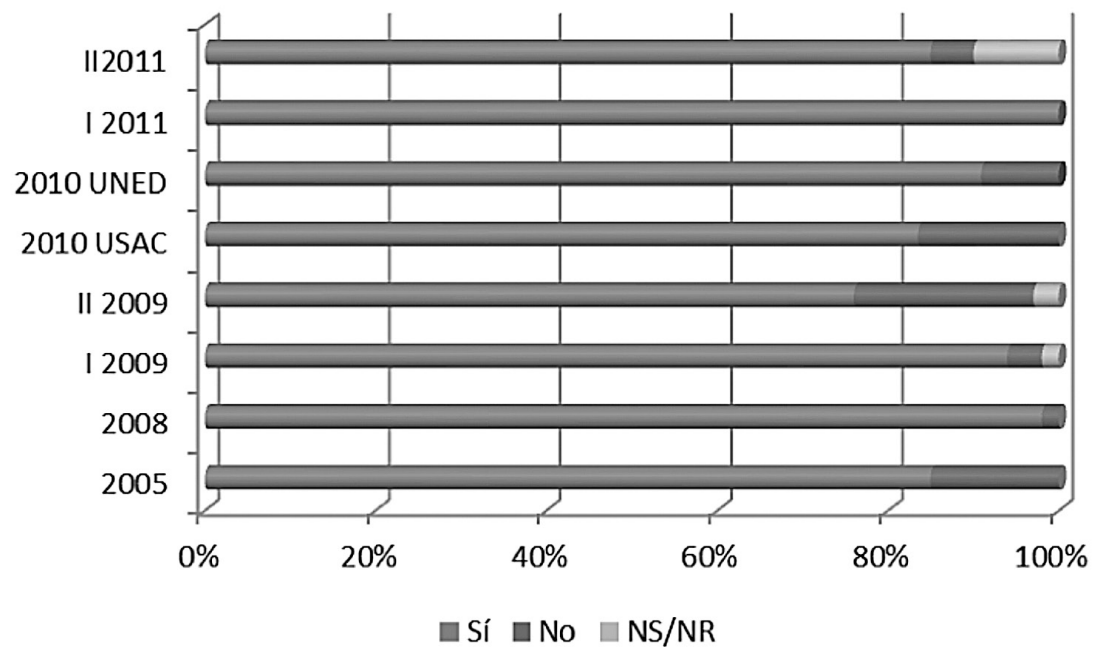

Figura 7. Evaluación de los participantes del proceso de discusión en las presentaciones de avance de tesis.

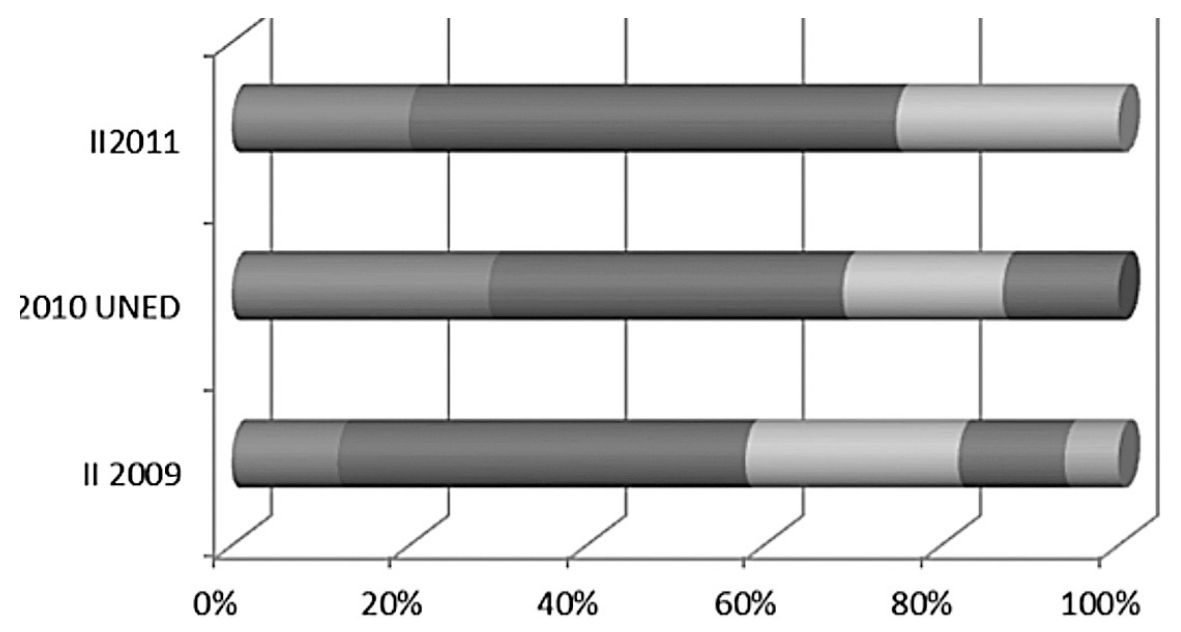

$\square$ Excelente $\square$ Muy Bueno $\square$ Bueno $\square$ Regular $\square$ Deficiente

programa, independientemente de la generación a la que pertenecen los estudiantes.

Sistemas de producción agrícola sostenible: "Estado actual y perspectivas para el desarrollo del cultivo de la mora en Costa Rica", "Adaptación y validación de sistemas de producción bajo ambiente controlado para cultivos hortícolas en la zona de San Carlos, Costa Rica", "Efectividad de extractos naturales en el manejo orgánico de la moniliasis del cacao en el estado de Chiapas, México", "El problema del mango niño en el mango-Manguifera indica L. Cv. Ataulfo en la región del Soconusco, Chiapas
México", "Efecto biocida de extractos de hojas y flores de Llama del bosque (Spathodea campanulata Beauv) en Guatemala".

Gestión de los recursos naturales: "Análisis histórico de las iniciativas de conservación del bosque tropical húmedo en las llanuras de la Cuenca del Río San Juan: hacia la creación de una reserva de la Biosfera", "Evaluación de la actividad antibiótica de nuevos compuestos naturales y su efecto sobre la morfología microscópica en México", "Gestión integral para el manejo sostenible de la cuenca hidrográfica del río Nizaito, Barahona, República Dominicana", 
"Propuesta metodológica para determinar cuál es el aporte del capital natural en las estrategias de vida de los pobladores de 2 sitios a partir de la experiencia del proyecto de equidad social a partir del conocimiento: Biodiversidad y comunidades de la UICN-INBio", "Función Ecosistémica de los Manglares Achaparrados Rhizophora mangle L. Río Sarstún, Caribe de Guatemala".

Gestión y cultura ambiental: "Desarrollo interdisciplinario de un proyecto educativo de orden ambiental en Escuelas de México", "Gestión del recurso hídrico en la Región Chorotega de Costa Rica: perspectiva de cooperación y conflicto entre autores", "Determinación de Helicobacter pylori en el abastecimiento de agua para consumo humano", "Síntesis de un material tipo hidrotalcita (ht) de composición mg-fe(iii) para la remoción de arsénico inorgánico total de fuentes de agua naturales", "Estimación Económico-Ecológica de la Deuda Ecológica de las Zonas Urbanas y Agrícolas Adyacentes a la Zona Protectora Cerros de La Carpintera en el Valle Central de Costa Rica".

Tecnologías electrónicas aplicadas: "Análisis de las interacciones dinámicas entre sistemas digitales y ecosistemas de cultivo de papa para el desarrollo de un modelo basado en su microclima", "Desarrollo de un protocolo de comunicaciones multifunción para redes de sensores para la protección ambiental en bosques costarricenses", "Red de sensores para detección temprana de incendios forestales en Costa Rica: aplicación en el Área de Conservación Guanacaste, Costa Rica", "Diseño de circuitos integrados CMOS aplicando optimización multiobejtivo".

\section{Bibliografía}

Comité de Gestión Académica. (2007). Informe de trabajo "Doctorado en Ciencias Naturales para el Desarrollo" al Consejo Nacional de rectores a dos años de trabajo. Febrero.

Comité de Gestión Académica. (2007). Informe de autoevaluación del "Doctorado en Ciencias Naturales para el Desarrollo". Universidad Nacional, Julio.

Comité de Gestión Académica. (2007). Informe de las actividades al Consejo de Escuela del Programa de Doctorado en Ciencias Naturales para el Desarrollo. Noviembre.

Consejo Nacional de Rectores (CONARE). (2003). Doctorado en Ciencias Naturales para el Desarrollo. Dictamen de Creación de la carrera. Diciembre.

Consejo Nacional de Rectores (CONARE). (2006). Estrategia siglo XXI: Conocimiento e innovación hacia el 2050 en Costa Rica. Marzo.

Guzmán Hernández, T.J. \& Victorino R.L. (2009). Doctorado en Ciencias Naturales para el Desarrollo: seguimiento y evaluación de sus primeras generaciones. En:Textual, No. 50, pp. 191-216. Universidad Autónoma Chapingo, México.

Guzmán Hernández T.J. (201 I). Informe de labores de las actividades del DOCINADE al Consejo de Escuela A. Noviembre.

Guzmán Hernández T.J (201 I). Informe de labores de las actividades del DOCINADE del 2005-2011 al Comité de Gestión Académica. Noviembre.

Partington, P. (1996). Desarrollo de la gestión y dirección para el personal académico en la educación superior. Formación y desarrollo para la Docencia y Gestión Universitaria. Cedecs Editorial, S.L.

Quintanilla, M.A. (1996). Nuevas ideas para la Universidad. En: Allen, J. y Morales, G. La Universidad del siglo XXI y su impacto social. Universidad Las Palmas de Gran Canaria.

Wharton, C. (1996). La educación avanzada en el siglo XXl Hace falta una academia global? En: Allen, J. y Morales, G. La Universidad del siglo XXI y su impacto social. Universidad Las Palmas de Gran Canaria. 